\title{
PROLIFERATIVE MURAL BRONCHIOLITIS
}

BY

\author{
ST. ENGEL, M.D., and G. H. NEWNS, M.D., M.R.C.P.
}

(From the Hospital for Sick Children, Great Ormond Street, London)

Bronchiolitis is a condition which occurs predominantly in infants and young children. Although the clinical picture and the grave significance are well known, the morbid anatomy and histology of the condition have been the subject of very little investigation. The anatomical features, as will be shown in a later paper, vary a great deal, there being a continuous gradation from the slightest to the severest forms. Two main groups must be distinguished: catarrhal and mural bronchiolitis. In the first type the mucous membrane alone is involved, in the second the whole wall is affected. Catarrhal bronchiolitis seems to be less common than mural bronchiolitis. The latter type of inflammation is the usual reaction of the bronchioles to infection.

Mural bronchiolitis appears in many degrees of intensity. It is useful to differentiate three groups: simple, proliferative and destructive mural bronchiolitis.

SIMPLE MURAL BRONCHIOLITIS means infiltration of the wall and engorgement of the vessels without any lesion or change in the epithelium.

In PROLIFERATIVE MURAL BRONCHIOLITIS infiltration and engorgement is ordinarily intense: In addition, proliferation or pathological growth of the epithelium, of the basal cells and, occasionally, of other elements of the bronchiolar wall or the surrounding lung tissue is characteristic.

The DESTRUCTIVE TYPE is marked by complete destruction of the epithelium and other adjacent constituents of the bronchiolar wall. In these cases the reaction of the surrounding lung tissue is generally more marked.

There is, apparently, no fundamental difference between the three types. It is a matter of degree, probably due to different infective agents or to secondary infections superimposed on the primary agent. Transitions from the one form to the other occur frequently. However, in most cases classification is possible.

This article deals exclusively with proliferative mural bronchiolitis (P.M.B.). This type shows the most characteristic features of the different degrees of mural bronchiolitis. It was first described (although it was called bronchopneumonia) in measles and later it was found also in whooping cough, diphtheria and influenza (Lange, 1901; Jochmann and Moltrecht, 1904; Hart, 1904; Huebschmann, 1917; MacCallum, 1919; Opie, 1921; Feyrter, 1925). Interest in P.M.B. was revived when American authors had to deal with large epidemics of measles and influenza in army camps (MacCallum, 1919; Opie, 1921). 
MacCallum has made a study of the pulmonary lesion in the case of measles in the adult and has published his results in a fine monograph. Later the pandemic of influenza produced numerous papers (Opie), showing that the same pulmonary lesion which MacCullum had seen in measles was a common sequel of influenza.

MacCallum gave the condition the name of ' interstitial bronchopneumonia,' observing that interstitial spread originates from the bronchioli. This interstitial spread is not, as will be pointed out later, an essential feature in infants. It will be shown that the condition is characterized rather by intense inflammatory changes throughout the whole bronchiolar wall. Pneumonia (interstitial or otherwise) may follow, but this is a secondary process. It seems advisable to keep these two processes separated.

This paper is only concerned with the primary lesion, which will be shown to be of the proliferative mural type, according to the classification set out above. The chief features of P.M.B. are characteristic. The wall is densely infiltrated with monocytes, a large proportion of which are plasma cells. The vessels of the adventitia are engorged. This infiltration and hyperaemia give rise to an enormous thickening of the bronchiolar wall. These features are common, more or less, to all forms of mural bronchiolitis. In the case of P.M.B. there are the proliferative features superadded which will be described in this paper.

At the time when MacCallum wrote his monograph (1919) no definite cause for 'interstitial bronchopneumonia' could be found. MacCallum himself came to the conclusion that the condition could be produced by various organisms, pneumococci, staphylococci and, above all, by haemolytic streptococci and the influenza bacillus. Our interest has been aroused by finding P.M.B. in different degrees of intensity in the lungs of a number of infants. None of these had measles or pertussis, nor could they be recognized clinically as influenza. There seemed to be only two possibilities: either they were cases of influenza without distinct clinical symptoms or they were some other condition, unknown up to the present. This might be an infection of unknown kind or a bronchiolar reaction to which no attention had been paid hitherto. A closer investigation appeared worthwhile.

\section{Case reports}

Case I. Female, aged two-and-a-half months. History of cough and vomiting of one day's duration. When seen, the infant was very ill and cyanosed. There were signs of consolidation present at the left base, and later consolidation at the right base. A temporary improvement followed treatment, but the infant died after an illness of three weeks. Diagnosis: bronchopneumonia. N.B.: x-ray showed consolidation of both lower lobes.

Post-MORTEM. Fibrinous pleurisy over both lower lobes. Thick yellow pus in the trachea and main bronchi. Typical appearance of bronchopneumonia in both lower lobes. B. Pfeiffer grown from the bronchial pus.

Case 2. Female, aged three months. The birth was normal. Four weeks afterwards the baby was observed to make a whistling noise on breathing. She was frequently cyanosed. She was admitted for investigation. Bronchitis 
developed. There was no pyrexia. The general condition became worse. Death occurred after an illness of three to four weeks. Diagnosis: Atelectasis of the left upper lobe.

POST-MORTEM. Thick yellow pus in the trachea and bronchi. Scattered areas of consolidation and collapse were present in the whole of the right lung and the left lower lobe.

Case 3. Male, aged nine months. Since the age of four months the baby had frequently vomited. There was only a slight gain in weight. He was admitted for investigation. The vomiting continued and the infant became dehydrated. A cough developed. Later signs of bronchopneumonia appeared. The infant died after an illness of two to three weeks.

POST-MORTEM. Both lower lobes were solid, due to a combination of collapse and broncho-pneumonic infiltration. The other lobes were overdistended.

Case 4. Female, aged thirteen months. This was a typical case of coeliac disease with characteristic fatty stools. Soon after admission, bronchopneumonia developed and the infant died after an illness of two weeks' duration.

POST-MORTEM. Pus was present in both middle ears. There was a confluent type of broncho-pneumonia in the left lower lobe and a patchy infiltration of all the other lobes.

Case 5. Female, aged eight months. The infant was a mongol. For several days she had had nasal catarrh. The day before admission she was feverish and started to cough. Dyspnoea was present. On admission, the infant was moribund. She was cyanosed and had grunting respirations. There were signs of consolidation in both lower lobes. Death took place shortly after admission. Diagnosis: broncho-pneumonia.

POST-MORTEM. Heart: there was a large deficiency in the inter-auricular septum; this was the only congenital lesion. Lungs: there were solid areas in both lower lobes and the apex of the right upper lobe.

\section{Clinical aspects}

It will be seen from the case reports that there is nothing in the clinical appearances of these cases to distinguish them from cases of broncho-pneumonia, whether of the primary type or of the kind following or complicating some preexisting disease. In two of the cases ( 1 and 5$)$ the onset was sudden and the patients became rapidly toxaemic, with severe respiratory embarrassment. The first case died after an illness of three weeks' duration, the latter after one of only two to three days. Cases 3 and 4 were infants who were extremely wasted, the one from persistent vomiting, the other from coeliac disease. They developed 'broncho-pneumonia' while in hospital and died after short illnesses of about two or three weeks' duration.

Case 2 was diagnosed as atelectasis. Although there was evidence of consolidation of the lung, there was no pyrexia at any time during the course of the illness. At autopsy, however, there was definite consolidation of both lungs associated with collapse.

\section{The histological picture}

The general impression of the microscopical picture is that of bronchiolitis combined with collapse. In view of the macroscopical appearance one would have expected to find broncho-pneumonic foci. However, the macroscopical 
appearance of broncho-pneumonia is only due to the enormous thickness of the inflamed wall.

The lung tissue in the area affected by bronchiolitis is, as a rule, collapsed, but the air-spaces are not infiltrated nor is the interstitia. Some desquamated cells are frequently seen within the air-spaces. Fibrin is absent.

Thickening of the bronchiolar wall is, in the first place, produced by dense infiltration with monocytes. Many of them are plasma cells. The blood vessels of the wall are strikingly engorged. This contributes a great deal to the thickening of the part of the bronchiolar wall external to the elastic layer.

The bronchiolar wall consists of two chief parts, which are separated by the elastic sheath external to the epithelium. There is, however, an almost imperceptible layer of cells interposed between the elastica and epithelium. It consists normally only of a few oblong, spindle-shaped cells; whether they form a true separate layer or whether they are part of the basal membrane will not be discussed in this paper. We will refer to this shortly as the 'basal layer' or ' basal cells.' These cells proliferate rapidly in our cases of bronchiolitis. They lose their fusiform shape and grow more roundish-oblong. Within a short time (a few days are sufficient), they may produce a many-layered stratum, lining the inside of the bronchiolar tube between the elastica and the epithelium. The layer is soon vascularized by ingrowth of vessels from the adventitia, which perforate the elastica.

The epithelium undergoes various changes. Sometimes it is loosened and cast off. In most cases this is not a post-mortem effect or an artefact produced by the technique of fixation and embedding as is frequently assumed. In one case we found epithelium aspirated into the air-spaces. Sometimes the epithelium was entirely lacking; sometimes it could be seen in aggregations in the lumen: neither appearance is compatible with the detachment from postmortem or from technical causes. A most peculiar feature is an abnormal growth of the epithelium which is frequently met with. The epithelial cells, which ordinarily in the smaller bronchioles are low and still more so in the respiratory bronchioles, assume a columnar shape of extraordinary height. The single layer which is present normally in the larger bronchioles is transformed into a kind of stratified epithelium. Between the epithelial cells more or less numerous cells can be seen migrating through to the lumen.

The lumen is more or less filled by cellular masses, monocytes and polynuclears in different proportion. The respiratory bronchioles are frequently filled by a more mucous content containing only a few cells.

The lung, as mentioned above, is as a rule not affected, or at least not at the beginning. As a consequence of the obstruction to the passage of the air, the lung tissue collapses. Since a collapsed lung is more liable to infiltration and inflammation, it is no wonder that pneumonia frequently develops. Pneumonia is not directly but only indirectly due to the bronchiolitis, the link between them being the collapse. The bronchiolar inflammation stops at the beginning of the air-spaces; the air spaces adjacent to the bronchiolar wall are not affected. Interstitial spread was not observed. Only in one case interstitial infiltration in a zone in the immediate vicinity of the bronchioli could be seen.

The lymphatic system is distinctly affected in the majority of cases. This is not peculiar, as lymphangitis occurs in numerous infections of the lung. Much more noteworthy are the widespread lymphatic nodules which were present in two cases. In one of those lymphangitis was also present. The miliary nodules are ordinarily attached to the bronchioles and less frequently to vessels. Sometimes they are at the usual place between bronchiole and pulmonary artery. The nodules often consist not only of an accumulation of lymph cells but are sometimes amply vascularized so that they resemble much 
more true lymph-glands. The two cases in which the nodules were observed were older infants, of six and thirteen months.

It is well known that the type of bronchiolitis described leads frequently to bronchiolitis obliterans. This in its earliest stage was seen in the youngest infant (ten weeks). The respiratory bronchioles, and these only, were filled with mucous-like more or less homogeneous masses, which projected somewhat into the air-spaces. In these masses young vessels filled with red corpuscles and also fibroblasts could be observed. The whole duration of the illness in this case did not amount to more than twelve to fourteen days.

\section{Discussion and conclusions}

In the present cases, as mentioned above, bronchiolitis was not associated with interstitial spread, in contrast to the findings of American authors in adults. McNeil (1929) in his investigations of pneumonia in children also observed no interstitial spread, while Lauche (1928) in his comprehensive article comes to the conclusion that, in this respect, there is a difference between adults and children. The cases described in this paper are also in no way associated with alveolar pneumonia. The primary lesion is bronchiolar and is a definite anatomical and histological entity. Hitherto the purely bronchiolar nature of this condition has not been recognized. In the literature it is referred to as bronchopneumonia. The reason for this confusion might be that the macroscopical appearance of multiple pulmonary foci and the histological picture of a patchy consolidation resembles broncho-pneumonia. These appearances are not, however, produced by peri-bronchiolar sheaths of infiltrated alveoli, but by an infiltration of the bronchiolar wall itself, which becomes greatly enlarged in consequence (fig. 1 and 2). In cases like those described by MacCallum the name ' interstitial broncho-pneumonia' is obviously justified, because there was a widespread interstitial process originating from the inflamed bronchioli.

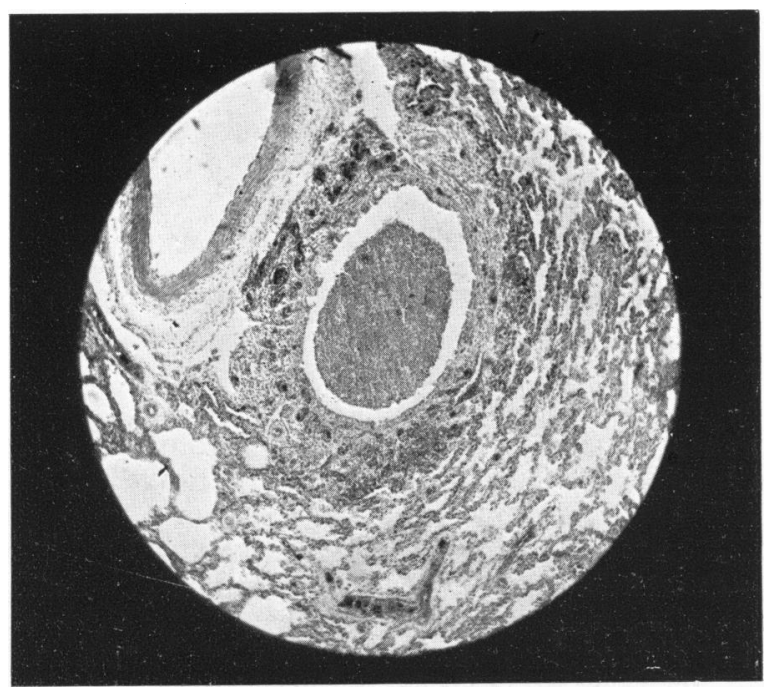

FIG. 1.-Proliferative mural bronchiolitis. Bronchiolus of $0.3 \mathrm{~mm}$. diam. ( $\times 30$, Case No. 2$)$. 


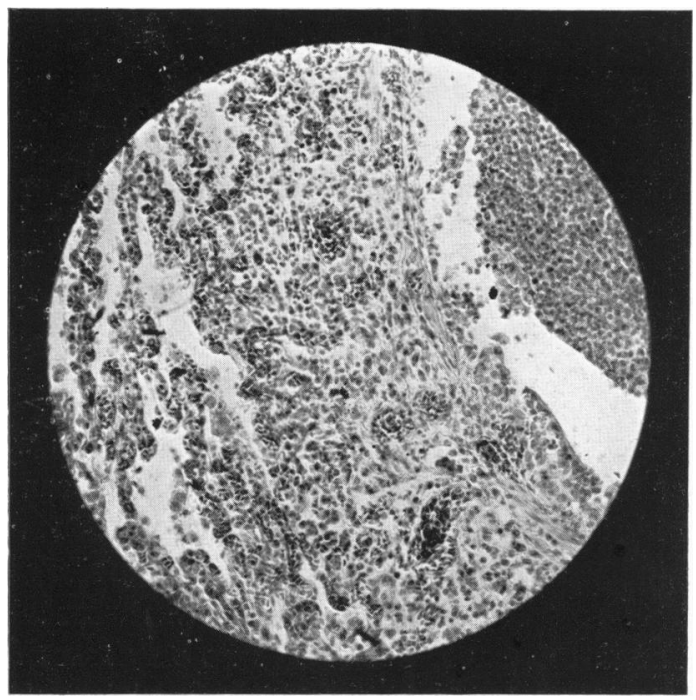

Fig. 2.-High-power view of part of fig. 1. $(\times 125$.

The characteristic thickening of the bronchiolar wall by hyperaemia and cellular infiltration has been described in measles and influenza many times before. McNeil and his co-workers recently referred to it. It is not necessary, therefore, to comment on its details. The proliferative changes, however, that we have described in the mucous membrane appear to be of the greatest importance. These changes are of a delicate nature and have apparently been overlooked up till now.

In order to understand these changes it seems useful to bear in mind the histological structure of the bronchiolar wall. As shown in fig. 3, 4 and 5, six components can be distinguished. Beginning from the lumen the sequence is as follows. First, columnar or cubical epithelium; second, the basal cells; third, the basal membrane; fourth, the elastic sheath; fifth, the musculature; and sixth, the adventitia. There is no tunica propria, as found in the bronchi. Of all these constituents, the basal cells, which are the least conspicuous, are the focus of interest. These cells are so small, so few and so hidden that, as a rule, they are not mentioned even by the few people who have dealt with the structure of the bronchiolar wall. Miller (1937) considers them to be analogous to the basal cells of the stratified epithelium of the trachea and the larger bronchi. Whether this is right will not be discussed in this paper.

In order to obtain a correct picture of the histological components of the bronchiolar wall it is most essential to use elastic tissue stain. In this way a clear differentiation between the mucous membrane and the rest of the bronchiolar wall is produced, as an elastic sheath forms the border between the mucous membrane and the external part of the wall. The elastica is composed mainly of fibrils running longitudinally. They are so close together that in cross sections they appear as a continuous ring when looked at with a low or a medium power. Between the elastica and the epithelium the basal cells can be observed as flat, spindle-shaped cells, scattered irregularly. They do not 

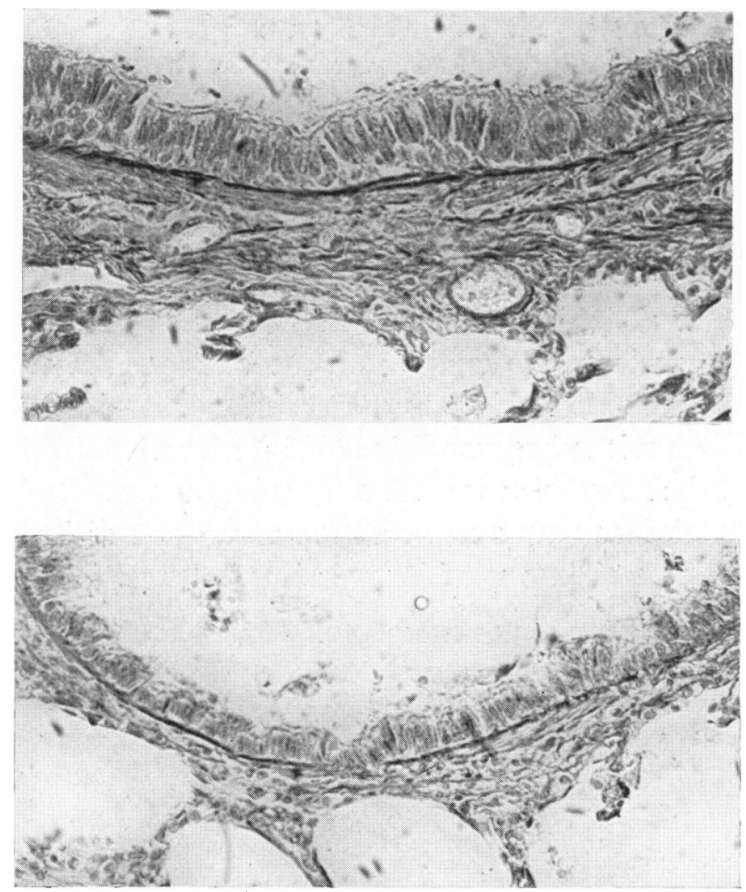

FIG. 3 and 4.-Cross section of the wall of bronchioli of 0.6 and $0.3 \mathrm{~mm}$. diameter respectively. From child aged five months. ( $\times 200$, cf. diagram fig. 5.)

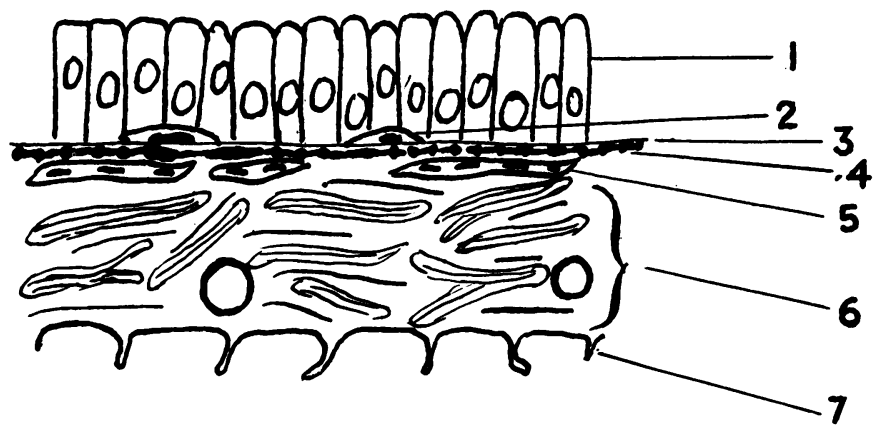

Fig. 5.-Diagram after Fig. 4, $\times 700$.

1. Ciliated epithelium. 2. Flat basal cells. 3. Basal membrane. 4. Elastic sheath 5. Muscles. 6. Adventitia composed of connective tissue, elastic fibres and vessels. 7. Alveolar walls.

(Bronchiolus 0.3, $\times 700$.) 


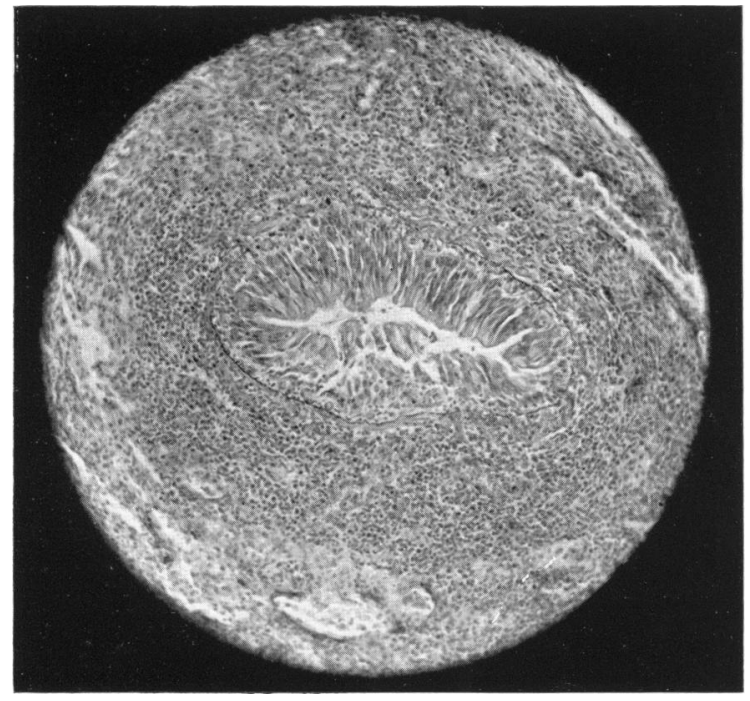

FIG. 6.-Bronchiolus of $0 \cdot 3 \mathrm{~mm}$. diameter, case No. 1, $\times 140$. Infiltration of the wall. Epithelium enlarged. Between epithelium and elastica proliferated basal cells.

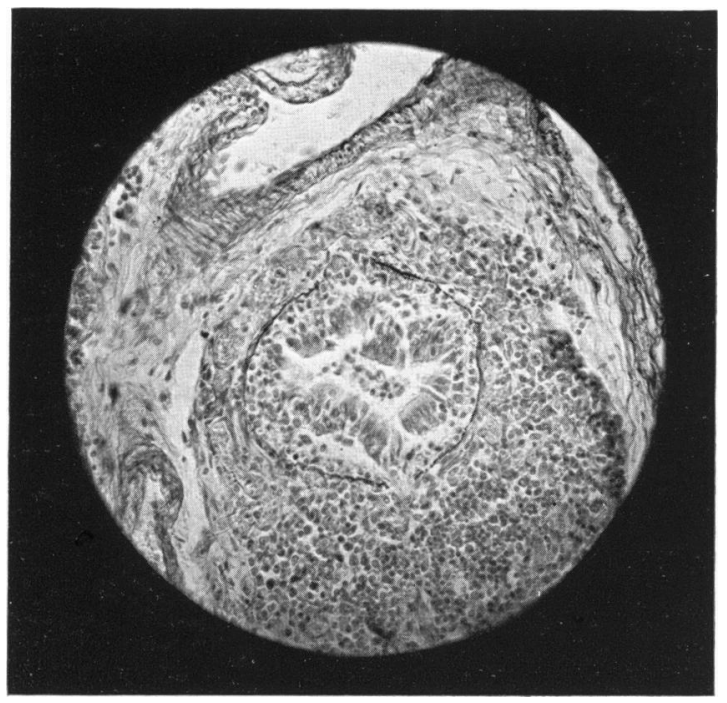

FIG. 7.-Bronchiolus terminalis, $0.18 \mathrm{~mm}$. diameter, case No. $1, \times 125$. Infiltration of the wall, engorgement of the vessels. Lymph nodule attached to the wall. Epithelium pushed to the centre of the lumen by proliferated basal cells. 
form a continuous ring. They are more likely to be seen in pathological cases such as those reproduced in the photomicrographs (fig. 6 and 7).

In our cases a surprising change in the basal cells was seen. They proliferated rapidly and formed within a few days a definite stratum between the elastica and the epithelium. The extent of the proliferation varied. Sometimes there was only a thin though quite obvious layer; sometimes it became so great that the lumen was visibly narrowed and the epithelium was pushed to the interior of the lumen (fig. 7). The enlarged basal layer can be seen in illustrations of different papers on pneumonia, bronchitis and interstitial broncho-pneumonia, but, surprisingly, no reference is made to it. Goldberger (1918) is the only one to describe the proliferation of the basal cells. This was in one case of diphtheria and he considered that it was rare and exceptional.

The proliferation occurred only in the bronchioles. Variations in the extent of the cellular growth depend on the size of the bronchioles, the age of the child and on several other factors which, however, cannot be discussed here in detail. The proliferative process does not occur where a marked basal membrane is present. Its formation depends on the age. In the newborn and the young infant only the larger bronchi are provided with a basal membrane which is visible in an ordinary histological preparation. Gradually it grows stronger within the subsequent months and, at the same time, descends to the smaller bronchi. In older children even the larger and the medium bronchioles ( 1.0 to $0.3 \mathrm{~mm}$.) are provided with an easily discernible basal membrane.

The question why mural bronchiolitis is sometimes a proliferative one and sometimes not will be briefly discussed. In the measles-influenza type proliferation is always present. In other less specific infections proliferation is absent. Difference in the intensity of inflammation or the presence of a specific irritant might be responsible for these differences. The nature of the infecting agent seems to be the important factor, since proliferation does not occur in nonspecific mural bronchiolitis even when of great intensity. Slight swelling of the basal cells which makes them more easily recognizable occurs in many cases of bronchiolitis. This cannot be confused with the abundant growth seen in our cases.

The proximity of the basal cells to the epithelium leads us to a further consideration of the epithelium. Sometimes it is merely dislodged from its base, in other cases it is completely separated. It might be found in garlands or coils somewhere in the lumen of the bronchiole. Occasionally it is surrounded by pus or mucus so that its displacement during life cannot be doubted. In still other cases no epithelium at all can be seen. If the elastic tissue is stained, it is seen that at the site of the mucosa there is nothing but a layer of proliferated basal cells.

Enlargement of the epithelium is another feature occurring in proliferative mural bronchiolitis. This is well known from the study of the lung in measles and influenza, and a great deal of discussion has taken place about its interpretation. Our contribution to this discussion is confined to one point. Is there any connexion between this hitherto unexplained growth of the epithelium 
and the proliferation of the basal cells? There appear to be only two possibilities. Either the growth of the epithelium and the basal cells is stimulated by one and the same irritant or, as the proliferation of the basal cells is more constant, this layer is primarily affected. This problem will be considered in detail in a later paper.

We return now to the problem of whether the characteristic features of proliferative mural bronchiolitis might be caused by some peculiarity of the infecting agent. The histological picture presented in this paper is, as mentioned above, almost identical with that occurring in measles, whooping cough and influenza. Our cases might be of influenzal origin. This must the more be taken into consideration as several authors have found the Pfeiffer bacillus in almost every case of bronchiolitis (Opie, 1921; Liston, 1929). On the other hand, one must consider the possibility of a particular agent or of a group of agents being the cause of the same type of bronchiolitis in measles, whooping cough, influenza, etc. These agents, according to modern ideas, might belong to the virus group. Influenza and measles are now considered to be virus diseases, and some bacteriologists consider that a virus may play a part in the etiology of pertussis.

McCordock (1933) produced a typical mural bronchiolitis of the measles type by the introduction of dilute vaccine virus into the trachea of rabbits. Broncho-pneumonia could also be produced by superimposition of infection by bacteria. In a recent paper (Goodpasture et al., 1939) several cases of mural bronchiolitis in children suffering from measles and whooping cough were described as 'virus pneumonia' because, histologically, inclusion bodies were found.

These facts, taken together, support the idea that virus infection may play an important part in P.M.B.

We.will conclude by saying there is no clear-cut clinical picture corresponding to the definite anatomico-histological appearance. The general clinical symptoms were those of an ordinary broncho-pneumonia. There was considerable respiratory distress, with dyspnoea and cyanosis. This is not to be wondered at when one examines the anatomical lesion. The obstruction of so many bronchioles and the consequent collapse of the air-spaces diminish the respiratory surface very considerably.

In cases of acute respiratory infections in infants, causing considerable respiratory embarrassment, the physician should bear in mind the possibility of bronchiolitis and not make a general diagnosis of broncho-pneumonia in all such cases.

\section{Summary}

1. Bronchiolitis can be classified into 'catarrhal' and 'mural' types. The latter must be subdivided into ' simple,' ' proliferative' and 'destructive' bronchiolitis.

2. Proliferative mural bronchiolitis is the type occurring in measles and influenza. We observed this type in several infants who did not apparently suffer from either of these infections. 
3. The gross change is an enormous thickening of the bronchiolar wall and collapse of the lung tissue.

4. This, and not peribronchiolar infiltration, is responsible for the macroscopical picture of ' disseminated broncho-pneumonia.'

5. The centre of the lesion is situated in the medium bronchioles. Larger bronchioles, especially when furnished with a definite basal membrane, and bronchi are much less affected. This applies to the infiltration of the wall as well as to the engorgement of the vessels.

6. The proliferative part of the lesion affects predominantly the basal cells and the epithelium.

7. True pneumonia may develop as a consequence of mural bronchiolitis, but this is a secondary process.

8. Many facts point to the possibility of virus infection as a cause of proliferative mural bronchiolitis.

9. The clinical picture in our cases was indefinite. The possibility that proliferative mural bronchiolitis might be a disease sui generis is put forward for discussion.

Thanks are due to the honorary staff of The Hospital for Sick Children for the opportunity to carry out this investigation and for permission to use the post-mortem and case records.

\section{REFERENCES}

Engel, St. (1935). Handbook of Children's Diseases, 3, ed. by Pfaundler and Schlossmann, London.

Feyrter, F. (1925). Virchows Arch., 255, 753.

Goldberger, F. (1918). Z Zbl. allg. Path. path. Anat., 29, 510.

Goodpasture, E. W., et al. (1939). Amer. J. Dis. Child., 57, 447.

Hart, C. (1904). Dtsch. Arch. klin. Med., 79, 108.

Huebschmann, P. (1917). Beitr. path. Anat., 63, 202.

Jochmann, G., and Moltrecht (1904). Ibid., 51, 251.

Laidlaw, P. P. (1935). Lancet, 1, 1118.

Lange, W. (1901). Dtsch. Arch. klin. Med., 70, 342.

Lauche, A. (1928). Handbuch Path. Anat., vol. 3, part I, 701.

Liston, W. G. (1929). Arch. Dis. Childh., 4, 283.

MacCallum, W. G. (1919). The Pathology of the Pneumonia in the United States Army Camps, New York.

McNeil, C. (1929). Arch. Dis. Childh., 4, 83, 111.

McCordock, H. A., and Muckenfuss, R. S. (1933). Amer. J. Path., 9, 221.

Miller, W. S. (1937). The Lung, Springfield.

Moncrieff, A. A. (1934). Diseases of Children, Ed. by Thursfield and Paterson, London, p. 424.

Opie, E. L., et al. (1921). Epidemic Respiratory Disease, St. Louis.

Policard, A. (1938). Le Poumon, Paris.

Straub, M. (1937). J. Path. Bact., 45, 75. 